\title{
Zur Palökologie der Euproopiden im Nordwestdeutschen Oberkarbon
}

\author{
Stephan Schultka ${ }^{1}$ \\ Mit 4 Abbildungen und 2 Tafeln
}

\begin{abstract}
Zusammenfassung
Tierische Fossilien aus dem Westfal D des Piesbergs bei Osnabrück sind seit 1860 vereinzelt immer wieder beschrieben worden. Doch erst durch die Funde der letzten 30 Jahre zählt der Piesberg zu den bedeutendsten Fundorten terrestrischer Fauna im Karbon. Erstmals werden 45 stratifiziert aufgesammelte Individuen von Euproops untersucht. Einige Details der Morphologie sowie eine dichte Behaarung werden beschrieben. Auf der Basis dieser Merkmale sowie der Verteilung juveniler und adulter Individuen im geologischen Profil wird eine Deutung des Lebensraumes der Euproopiden versucht. Danach hielten sich die juvenilen Individuen dieser Tiergruppe als Laubstreubewohner in feuchten Spülsäumen flacher ruhiger Gewässer und Laubstreuhorizonten auf. Die adulten Formen lebten vornehmlich benthisch in ufernahen Gewässern und verließen wohl nur noch gelegentlich das Wasser.
\end{abstract}

Schlïsselwörter: Westfal D, Nordwestdeutschland, Piesberg, Xiphosura, Euproops, Paläoökologie.

\begin{abstract}
Since 1860 articulated fossils of terrestrial taxa have been described from lower Westfalian D sediments of the Piesberg section near Osnabrück (northwestern Germany). In this paper 45 individuals of the genus Euproops, sampled from particular levels in the profile, are reported on for the first time. Analysis of the fossil material reveals new morphological details especially setae densely covering the ventral side - that, combined with the geological setting of the profile, gives novel insights into the palaeoenvironment and to the hypothesis of subaerial activity. Young individuals of Euproops may have been "litter dwellers" inhabiting forest-litter and partly decayed plant material that had washed ashore. By contrast, adults formed a principal component of the nearshore benthic fauna. They were able to leave the water, but only for short periods. This is proved by the occurence of adults and juveniles in different lithologies.
\end{abstract}

Key words: Westphalian D, north-western Germany, Piesberg, Xiphosura, Euproops, palaeoecology.

\section{Einführung}

Die drei Karbon-Aufbrüche im nordwestdeutschen Raum - der Schafberg bei Ibbenbüren sowie der Hüggel und der Piesberg bei Osnabrück (Abb. 1) sind bereits seit alters her geologisch wie paläobotanisch eingehend bearbeitet worden (u. a. Hoffmann 1826, Roemer 1860, Gothan 1925, Bode 1927, Josten 1966, Maithy 1972). Nur wenige Arbeiten befassten sich bis zum Beginn der 70er-Jahre mit paläozoologischen Funden (Bölsche 1875, Knauff et al. 1971, Siegfried 1972), was auf die geringe Funddichte zurückzuführen war. In den letzten 30 Jahren konnte aber eine sehr individuen- und artenreiche Fauna geborgen werden, wodurch insbesondere der Pies- berg heute zu den bedeutendsten Fundorten für terrestrische Arthropoden des Karbons in Europa und vielleicht sogar in der Welt gehören dürfte. Publiziert wurden vornehmlich Insektenfunde (Brauckmann 1983, 1991, 1995, Brauckmann \& Herd 2000), die Bivalven wurden erstmals von Sorusch (1981), Schultka (1988) und Eagar et al. (1998) erwähnt.

Seit 1875 ist das Vorkommen von Euproopiden im Piesberg bekannt (Bölsche 1875) und auch in der Folgezeit wurden ganz vereinzelt Funde von dieser Tiergruppe gemacht (Boekschooten 1968, Brauckmann 1982). Sie repräsentieren im Oberkarbon eine wichtige Gruppe im Ökosystem, wie ihr häufiges Auftreten im gesamten euramerischen Raum belegt (Copeland

\footnotetext{
${ }^{1}$ Museum für Naturkunde, Institut für Paläontologie, Invalidenstraße 43, D-10115 Berlin, Germany. e-mail: stephan.schultka@rz.hu-berlin.de Erhalten April 2000, angenommen Mai 2000
} 
1957, Fisher 1979. Schultka 1988, Todd 1991. Anderson 1994, 1997).

Das Hauptmerkmal der Euproopiden - wodurch sie sich grundsätzlich von den Bellinuriden unterscheiden - ist eine beginnende Verschmelzung der Pleuren des Ophistosomas. Diese Verschmelzung führt zur Bildung eines Dorsalpanzers, der bei einigen Genera kaum noch die ursprünglichen Pleuren erkennen lässt, wie z. B. bei den Genera Pringlia (Raymond) oder Liomesaspis Raymond (Schultka 1988, Anderson 1997). wobei Letztere ebenfalls vom Piesberg nachgewiesen ist. Die Gruppe bildet einen Nebenzweig der hauptsächlich marinen Entwicklungslinien der Xiphosura (Stœrmer 1952), der offensichtlich bereits im Übergang Paläozoikum/ Mesozoikum ausstirbt. Sie weisen im Vergleich zur Hauptentwicklungslinie - Neolimulus, $\mathrm{Pa}$ laeolimulus, Mesolimulus bis zum rezenten Limulus bzw. Tachypleus (Stœermer 1952) - Zwergwuchs auf.

\section{Material}

Dieser Arbeit liegen insgesamt 45 Euproopiden-Resten aus dem Profil der im Piesberg aufgeschlossenen Schichtenfolge zu Grunde (Abb. 2). Im Museum für Naturkunde zu Berlin (MB) befinden sich 7 Exemplare (Inv.-Nr. MB. A 1034-1040). während $31 \mathrm{im}$ Museum des Geologisch-Paläontologischen Instituts Münster (GPIM. ohne Nummern) aufbewahrt werden. Die restlichen 7 Exemplare stammen aus Privatsammlungen.

Im Hangenden des Flözes Zwei-Bänke, das mit dem Flöz Dickenberg am Schafberg bei Ibbenbüren korreliert wird und als die Basis des Westfal D im nordwestdeutschen Raum angesehen wird (Bässler et al. 1971). konnten 20 Euproopiden geborgen werden. Weitere 12 Exemplare stammen aus den Schichten über Flöz. Drei-Bänke und jeweils 2 bzw. 11 Exemplare aus dem Hangenden der Flöze Mittel und Itterbeck.

Zusätzlich wurden fünf Reste aus dem Karbon des Schafbergs bei Ibbenbüren. die umfangreiche Xiphosuren-Sammlung van der Heide's (Geologisches Museum. Heerlen. Niederlande) aus dem Westfal des Limburger Raumes. sowie die Exemplare in der Sammlung des Deutschen Bergbaumuseums Bochum (DMT) für vergleichende Beobachtungen herangezogen.

\section{Erhaltung}

Generell ist es sehr schwierig zu entscheiden, ob eine Exuvie oder eine Leiche vorliegt. Das liegt vor allem daran, dass bei der Häutung die Marginalsutur aufreißt und sich nach dem Herausschlüpfen des Tieres weitgehend wieder schlieBen kann (Stœrmer 1955; Schäfer 1964). Als Leichen wurden bei der Bearbeitung daher nur solche Funde angesehen, die schwache oder gar keine Verfältelungen zeigen und zumindest teilweise körperlich im Sediment eingebettet sind. Bei diesen Stücken ist nicht nur die Wölbung des Cephalothorax erkennbar, sondern darüber hinaus ragen die aufgestellten Genalstacheln in das Sediment und reißen beim Spalten des Gesteins ab. Sie sind aber im Gegendruck präparativ nachweisbar, was auch Anderson (1994) von Xiphosuren mit weitgehend körperlicher Erhaltung vor allem aus Sideritkonkretionen beschreibt. Anderson (1994) geht auf weitere taphonomisch bedingte Merkmalsänderungen ausführlich ein. Seine Beobachtungen stimmen vollständig mit den am Piesberg gewonnenen Erkenntnissen überein.

Davon ausgehend konnten lediglich drei Individuen eindeutig als Leichen angesprochen werden, alle anderen Reste dürften Exuvien angehören. Insgesamt 11 Stücke sind weitgehend vollständig erhalten, wobei allerdings nur drei Stücke noch Teile des Telsons tragen. Häufig konnten auch noch anhängende Teile der Extremitäten nachgewiesen werden, was gegen einen Transport der Exuvien spricht (Todd 1991, Anderson 1997).

Die Größen der einzelnen Individuen variieren sehr stark. Die größte Prosoma-Breite konnte mit $51 \mathrm{~mm}$ gemessen werden (das dazugehörige Opisthosoma war nicht mehr erhalten; Taf. 1: Fig. 3). Das kleinste Tier misst vom Prosoma-Vorderrand bis zum Ansatz des Telsons etwas mehr als $3,5 \mathrm{~mm}$, bei einer Breite des Prosomas von etwa 4,5 mm (Taf. 2: Figs 1, 2). Die morphologischen Merkmale bleiben bei diesen

Tafel 1. 1. Euproops rotundatus. Flöz. Zweibänke. mit breitem Randsaum, unter dem Prosoma ein Schreitbein hervorschauend (Pfeil): 2:1 (GPIM). 2. Ausschnitt aus 1. "Endschere". äußerer Scherenteil beweglich gegen den starren inneren; 16:1. 3, Euproops sp. Flöz. Zweibänke. größtes bisher bekanntes Prosoma: $2: 1$ (MB. A 1034). 4, Euproops rotundatus, Flöz Zweibänke. mit fast vollständig erhaltenem Telson: 2:1 (GPIM). 5. Euproops sp.. Flöz Zweibänke. Genalstachel mit daneben herausschauendem Endglied eines "pushers" (Extremität VI) mit kleiner Endschere ("pincer"): 8:1 (MB. A 1040)

Plate 1. 1. Eliproops rotundatus. Zweibanke coal seam. broad rim of opithosoma, one of the legs (extremities II-V) is to see on the right site of prosoma (arrowed): 2:1 (GPIM). 2. Part of 1. claw at the end of the leg, outer part of claw movable; 16:1. 3. Euproops sp.. Zweibänke coal seam. biggest prosoma still known up to now: $2: 1$ (MB. A 1034). 4. Euproops rotundatus. Zweibänke coal seam, with nearly complete telson: 2:1 (GPIM). 5. Euproops sp., Zweibänke coal seam, genal spine with last articulation of "pusher" (extremity VI) with little claw ( "pincer"): 8:1 (MB. A 1040) 


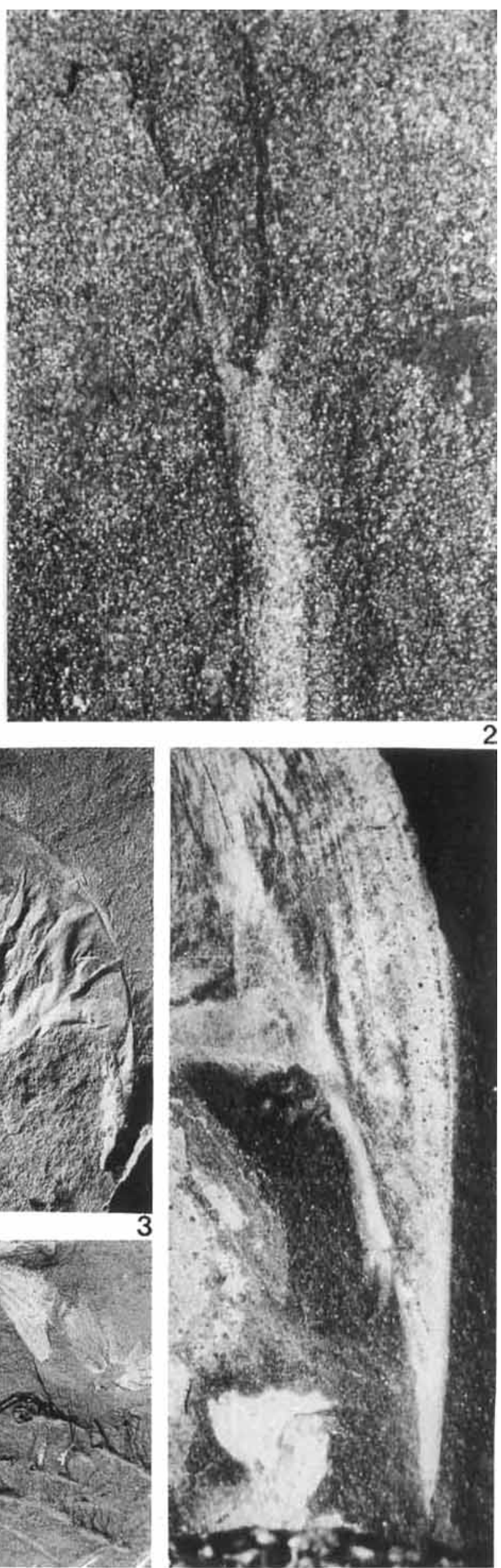

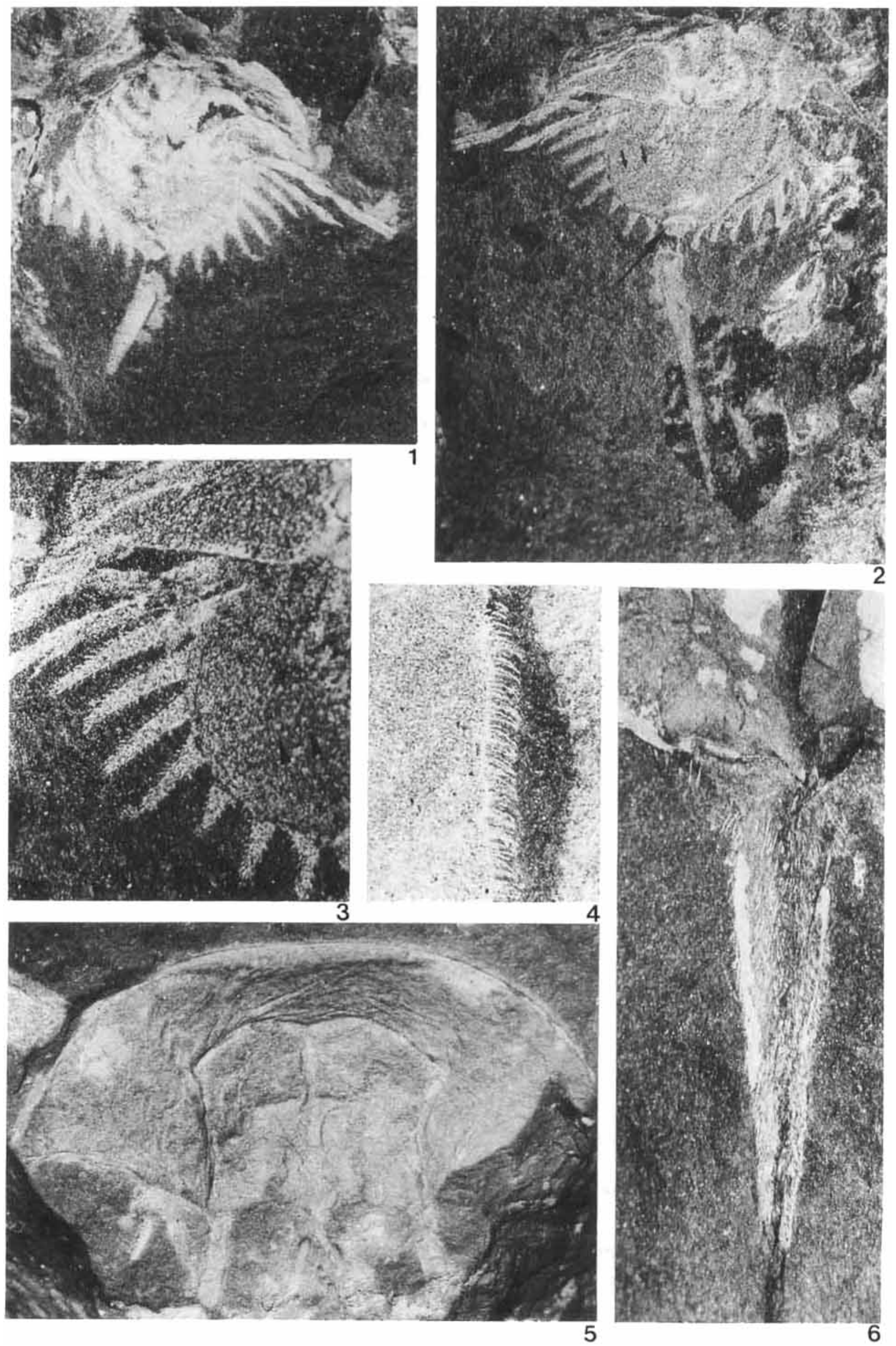
Individuen unterschiedlicher Größe weitgehend gleich, wie das auch bei der ontogenetischen Reihe der rezenten Limuliden festgestellt werden kann. Das einzige, deutliche Unterscheidungsmerkmal, das nicht auf Erhaltungszustände zurückgeführt werden kann, ist das bei allen Stücken gleichermaßen erkennbare Fehlen eines Randsaumes bei den sehr kleinen Exemplaren (Taf. 2: Fig. 3). Dadurch treten die Seitenstacheln deutlicher hervor, was möglicherweise zu der Auffassung führte, dass die juvenilen Formen dichter mit Stacheln besetzt seien als die adulten (Stœrmer 1955).

Der Randsaum scheint sich erst bei den ausgewachseneren Individuen $\mathrm{zu}$ entwickeln, wie das Übergangsformen auch belegen. Es treten nämlich mehrere mittelgroße Individuen auf, die einen Randsaum aufweisen, der aber im Verhältnis zur Gesamtbreite des Opisthosomas noch nicht die Breite der adulten Formen erreicht hat.

\section{Vorkommen}

Wie allgemein bei den Sedimentabfolgen im höheren Oberkarbon des Ibbenbüren-Osnabrücker Raumes nachweisbar ist, zeigt das Gesamtprofil des Piesbergs keine Zyklothem-Bildung im klassischen Sinne, wohl aber verschieden differenzierte ,fining- bzw. coarsening-upward"-Sequenzen (David 1987). Bedingt durch die Aufschlussverhältnisse in den 80er-Jahren wurden jeweils über dem tiefsten (Zweibänke) und dem höchsten aufgeschlossenen Flöz (Itterbeck) detaillierte Profile aufgenommen, die jeweils eine kleine Euproopiden-Population geliefert haben (Abb. 2). Die Euproopiden sind mit den typischen Vertretern der karbonischen terrestren Lebewelt wie Arthropoden, Süßwassermuscheln und seltener auch Fischresten vergesellschaftet. So konnten bisher am Piesberg Aphantomartus areolatus Pocock, Amynilyspes sp., Acantherpestes sp., Arthropleura armata Jordan, Blattodeen und andere Insektenreste, Anthraconauta tenuis (Davis \& Trueman), Palaeoxyris sp., Palaeoniscidenschuppen und ein Vertebraten-Zahn nachgewiesen werden (Brauckmann 1982, 1983, 1991, 1995, Brauckmann et al. 1997, Sorusch 1981, Schultka 1988). Zumindest die Euproopiden finden sich nur in sehr begrenzten Sedimentabschnitten, die ganz bestimmte Umweltbedingungen zu reflektieren scheinen.

Die mit den juvenilen Euproopiden vergesellschafteten Pflanzenreste sind generell unsortiert und meist schlecht erhalten bzw. auffällig stark mit Eisenhydroxiden imprägniert. Das spricht für eine sehr geringe Sedimentationsrate, wodurch es zu einem stärkeren Abbau der Pflanzenreste vor der endgültigen Fossilisierung kommen konnte. Calamitenwurzeln in situ sind relativ häufig. Nur selten finden sich in diesen Schichtabschnitten auch Reste von adulten Individuen. Diese treten bevorzugt in Sedimentbereichen auf, die weniger aber besser erhaltene Pflanzenreste und eine geringere Dichte von Calamitenwurzeln in situ aufweisen. Das belegt eine schnellere Sedimentation, wie das Todd (1991) für seine „sublithology $A$ " annimmt. Er vergleicht diese lithologische Einheit mit der Faziesassoziation 1A von Scott (1979). Die Wurzeln durchstoßen oder verletzten die Exuvien (Leichen) nicht.

\section{Bemerkungen zur Systematik}

Ausführlich belegt Anderson (1994), dass die meisten Arten der Gattung Euproops Meek durch Merkmale charakterisiert werden, die auf taphonomische Erscheinungen zurückgeführt werden können. Darauf basierend zieht er eine Reihe von Arten ein, lässt aber E. rotundatus (Prestwich) bestehen. Aufgrund fehlender siche-

Tafel 2. 1, Euproops cf. rotundatus, juvenil, mit der Ventralseite nach oben eingebettet, Flöz Zweibänke, 5 Beinpaare im Bereich des Prosomas erkennbar; 12:1 (GPIM). 2, Gegendruck von 1, mit zwei über das Prosoma hinausragenden Beingliedern (Extremität V und VI), Gelenkung an der Telsonwurzel gut erkennbar (Pfeil), Pleuralnähte mit Knoten besetzt (kleine Pfeile); 12:1 (MB. A 1037). 3, Ausschnitt aus 1, kräftige Beborstung der Randsaumstacheln, Pleuralnähte mit Knoten besetzt (Pfeil); 30:1 (GPIM). 4, Euproops sp., Flöz Zweibänke, Rand eines Genalstachels mit haarähnlichen Borsten; 30:1 (GPIM). 5, Euproops sp., juvenil (älteres, ontogentisches Stadium als 1), Flöz Dreibänke; 10:1 (MB. A 1039). 6, Euproops sp., Genalstachel eines adulten Tieres, ventral mit Beborstung, Flöz Zweibänke: 16:1 (MB. A 1035)

Plate 2. 1, 2, Euproops cf. rotundatus, juvenil, part and counterpart, Zweibänke coal seam, ventral side up, some legs are well seen, two legs projecting under the prosoma (extremities V und VI), well developed articulation of telson (arrowed), knots on pleural rims (minor arrows); 12:1 (1: GPIM, 2: MB. A 1037). 3, Part of 1, robust setae of prosomal spines, knots on the pleural rims (arrowed); 30:1 (GPIM). 4, Euproops sp., adult, Zweibänke coal seam, outer border of genal spine with hairlike setae; 30:1 (GPIM). 5, Euproops sp., juvenil (older ontogenetical stage than 1), Dreibänke coal seam; 10:1 (MB. A 1039). 6. Euproops sp., adult, Zweibänke coal seam, ventral side of genal spine with robust setae: $16: 1$ (MB. A 1035) 


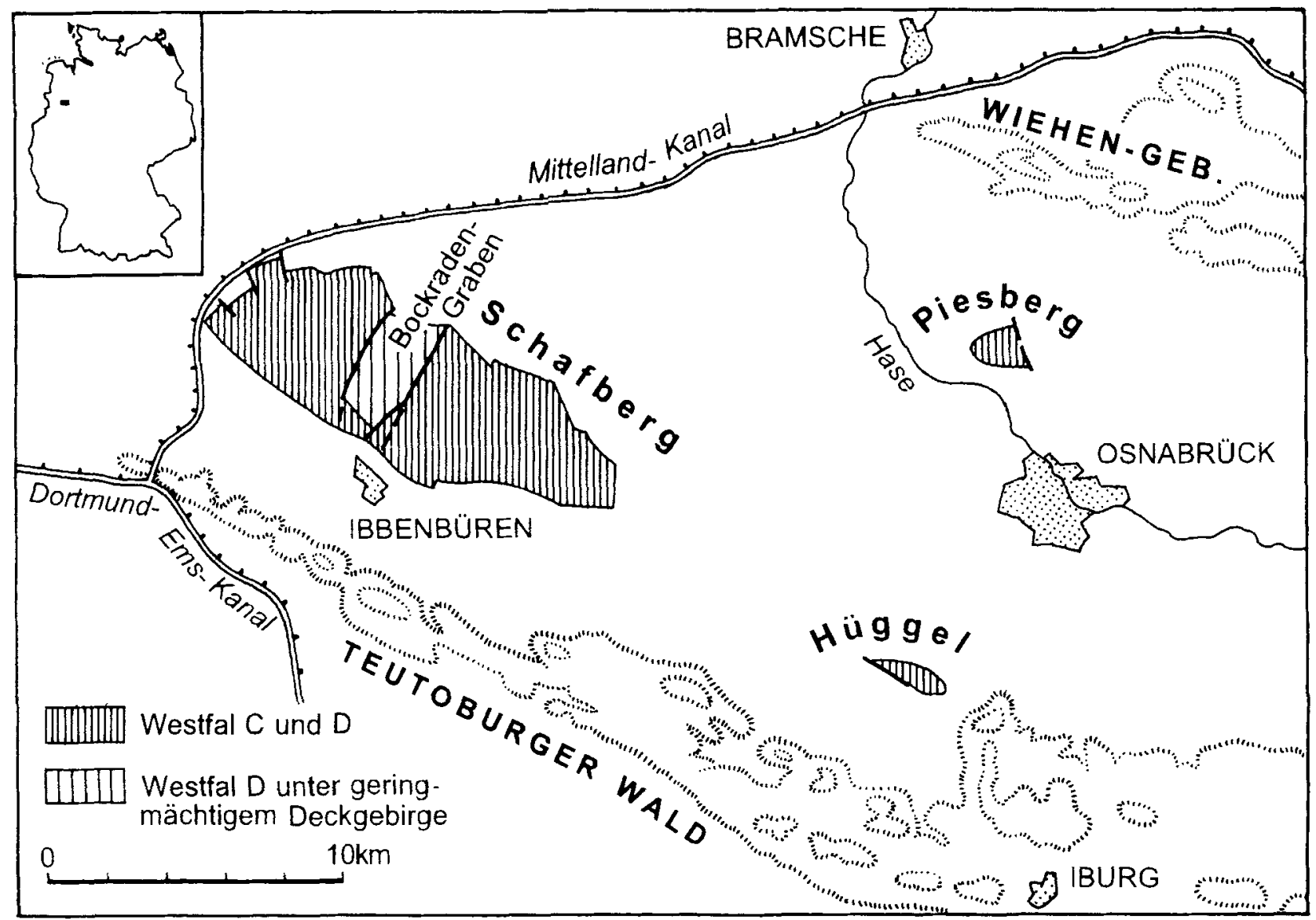

Abb. 1. Die drei Karbonaufbrüche im Osnabrücker Bergland (nach Bässler et al. 1971)

Fig. 1. Carboniferous deposits near Osnabrück. northwestern Germany (from Bässler et al. 1971)

rer Unterscheidungsmerkmale spricht Schultka (1988) vom "E. danae-E. rotundatus-Komplex" und weist darauf hin. dass die weit verbreitete Art E. rotundatus im gesamten amerikanischen Oberkarbon nicht vorkommt, E. danae (Meek \& Worthen) dagegen aber recht häufig. Dies ist umso auffälliger, wenn man berücksichtigt, dass ansonsten sehr wohl eine durchgehend gleichartige Entwicklung der Lebewelt des Oberkarbons im gesamten amerosinischen Bereich angenommen werden darf (Remy 1975).

Anderson (1997) unterscheidet nur noch $3 \mathrm{Ar}$ ten der Gattung Euproops, nämlich $E$. danae, E. anthrax (Prestwich) und E. rotundatus. Die wichtigsten Unterscheidungsmerkmale von $E$. rotundatus zu den beiden anderen Arten sind die auffällig langen Genalstacheln, der breite Opisthosomalsaum (und die daraus resultierenden relativ kurzen "Opisthosomalstacheln") sowie ein etwas breiter auseinander gezogenes Prosoma. Diese Unterscheidungsmerkmale sind gerade bei verdrückten Erhaltungszuständen nur schwer bewertbar (Anderson 1994). Da die Genalstacheln sowie die Intergenalstacheln leicht nach oben abgespreizt waren oder zumindest abgespreizt wer- den konnten, sind sie zudem oftmals stark verdrückt oder vollständig abgerissen und damit nur selten erhalten.

Wie das vom Piesberg gesammelte Material zu zeigen scheint, könnte die Breite des Randsaumes von ontogenetischen Stadien abhängig sein, was aber noch näher zu untersuchen wäre. Eine vollkommen klare Trennung der beiden Arten $E$. danae und $E$. rotundatus erscheint beim augenblicklichen Stand der Beschreibungen somit nicht möglich.

Wie bereits erwähnt, liegen keine vollständigen Individuen vor, zumindest Teile des Telsons fehlen. Damit kann auch nicht mit ausreichender Sicherheit gesagt werden, ob das relativ kurze Telson bei $E$. danae und $E$. anthrax im Vergleich zu dem langen Telson bei E. rotundatus (Taf. 1, Fig. 4) ein Unterscheidungsmerkmal darstellt. Bei den Individuen der rezenten Gattungen variiert die Länge des Telsons so stark, dass daraus kein taxonomisches Merkmal auf Artebene abgeleitet werden kann (mündl. Mitt. Anderson, Aberdeen, 2000). Allerdings vermutet Anderson (1997), dass ein kurzes, dickes Telson ein plesiomorphes Merkmal der Synziphosurinen im Ver- 


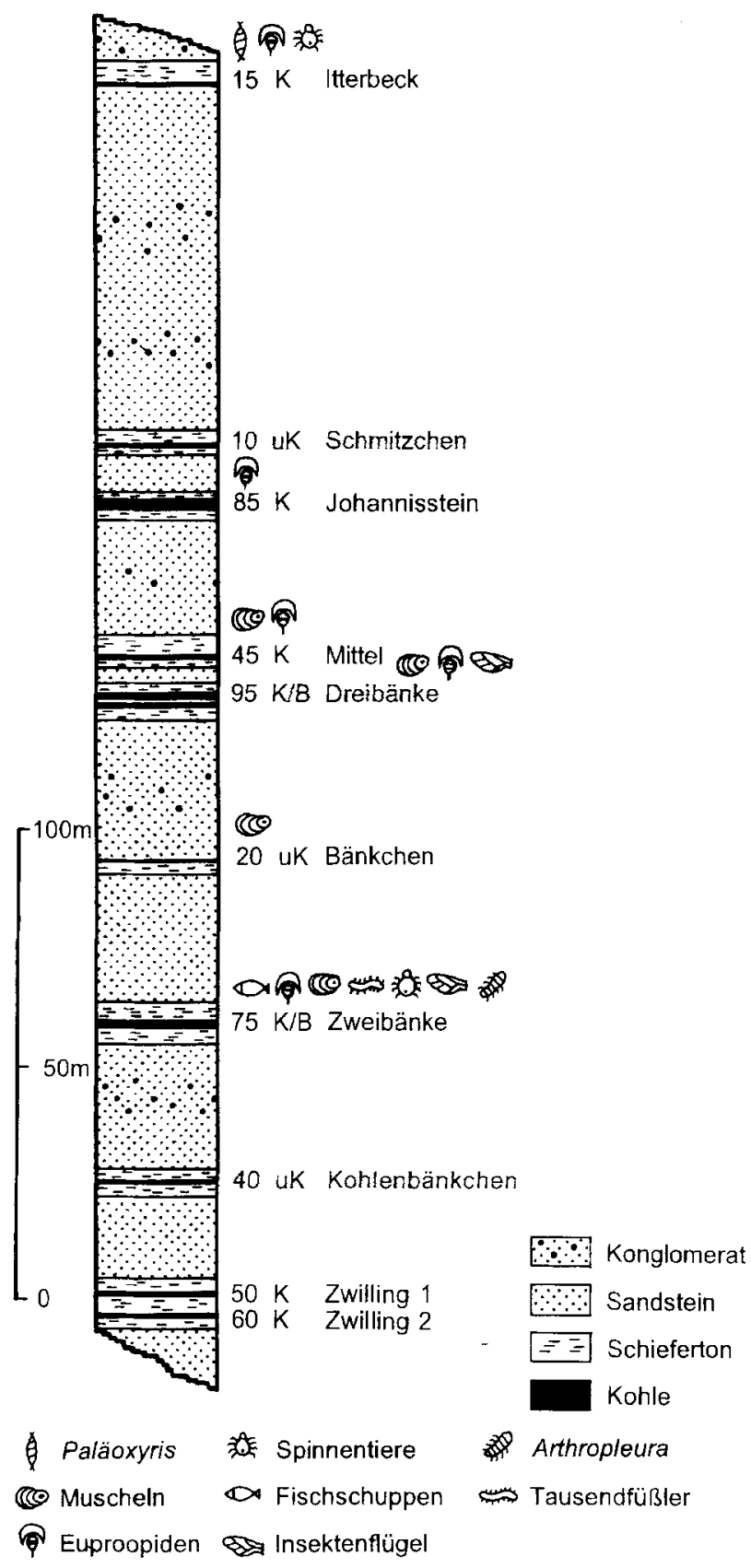

Abb. 2. Das aufgeschlossene und unter Flöz Zweibänke erbohrte Profil vom Piesberg bei Osnabrück mit der Faunenführung

Fig. 2. Distribution of faunistic elements in the profil of the Piesberg near Osnabrück

gleich zu den anderen Xiphosura ist. Er beschreibt eine Längenzunahme des Telsons in Abhängigkeit von der zunehmenden Verschmelzung der opisthosomalen Segmente. Bei seiner Rekonstruktion von E. danae (Anderson 1994) beträgt das Verhältnis Prosoma/Opisthosoma zu Telson etwa 1:1. Dagegen treten bei den Individuen vom Piesberg Verhältnisse größer als 1:1,5 auf, wobei ein durchschnittliches Verhältnis von $1: 2$ wie bei den Bellinuriden (Schultka 1994) angenommen werden kann.
Einige der größeren Euproops-Individuen tragen auf dem Opisthosoma kleine Knoten, die bei den Exemplaren vom Piesberg auf die Pleuralnähte konzentriert sind. Bei dem reichhaltigen Material der Sammlung van der Heide aus dem Westfal A und B des Limburger Oberkarbons findet sich im Gegensatz dazu diese Ornamentierung über beide Pleuralfelder gleichmäßig verteilt. Dieses Merkmal ist nur bei adulten Exemplaren gut zu erkennen, bei juvenilen Stadien sind solche feinen Merkmale entweder aufgrund der Erhaltung nicht mehr vorhanden oder nur schwer erkennbar (Taf. 2, Figs 2, 3). Bei den adulten Exemplaren finden sich häufig auch bei guter Erhaltung keine Ornamentierungen.

Das Auftreten ornamentierter und nicht ornamentierter Individuen in einem Schichtverband legt die Vermutung nahe, dass es sich hier um einen Sexualdimorphismus handeln könnte (Schultka 1988, Anderson 1997).

\section{Extremitäten}

Schon 1865 weisen Meek \& Worthen bei Euproops danae (damals noch als Bellinurus danae beschrieben) auf eine erhaltene Extremität hin, ohne klären zu können, zu welchem Beinpaartyp sie gehört. Das Bein schaut nur etwa $3 \mathrm{~mm}$ unter dem Prosoma hervor, paust sich aber noch durch den Panzer des Tieres durch. Diese Art der Erhaltung ist auch vom Piesberg bekannt (Taf. 1, Fig. 1). Ausführlich befasst sich Fisher (1979) mit dem Bau der Extremitäten, der dem der rezenten Limuliden sehr ähnlich ist.

Bei vielen Euproopiden-Resten vom Piesberg sind die Extremitäten zwar offensichtlich noch vorhanden, sind aber nur dann im Detail deutlich erkennbar, wenn sie über den Rand des Prosomas hinausschauen. Nachweisbar sind Schreitbeine (Extremitätenpaare II bis V; Abb. 3 rechts) sowie zweimal je ein „pusher“ (Extremitätenpaar VI; Abb. 3 links). Das zu Cheliceren umgewandelte Extremitätenpaar I konnte bisher nicht eindeutig nachgewiesen werden.

Wie bereits Meek \& Worthen (1865) messen konnten, erreichen die einzelnen Beinglieder annähernd $1 \mathrm{~mm}$ Breite, was im Verhältnis zur Länge recht schmal ist. So weist auch Anderson (1997) auf die auffällig schlanken Glieder der Extremitäten im Vergleich zu rezentem Material hin. Die Extremitäten von Carcinoscorpius (= Tachypleus) rotundicauda (Latrielle) (der karbonischen Limuliden besonders vergleichbar sein soll) haben ein Längen-Breiten-Verhältnis von 


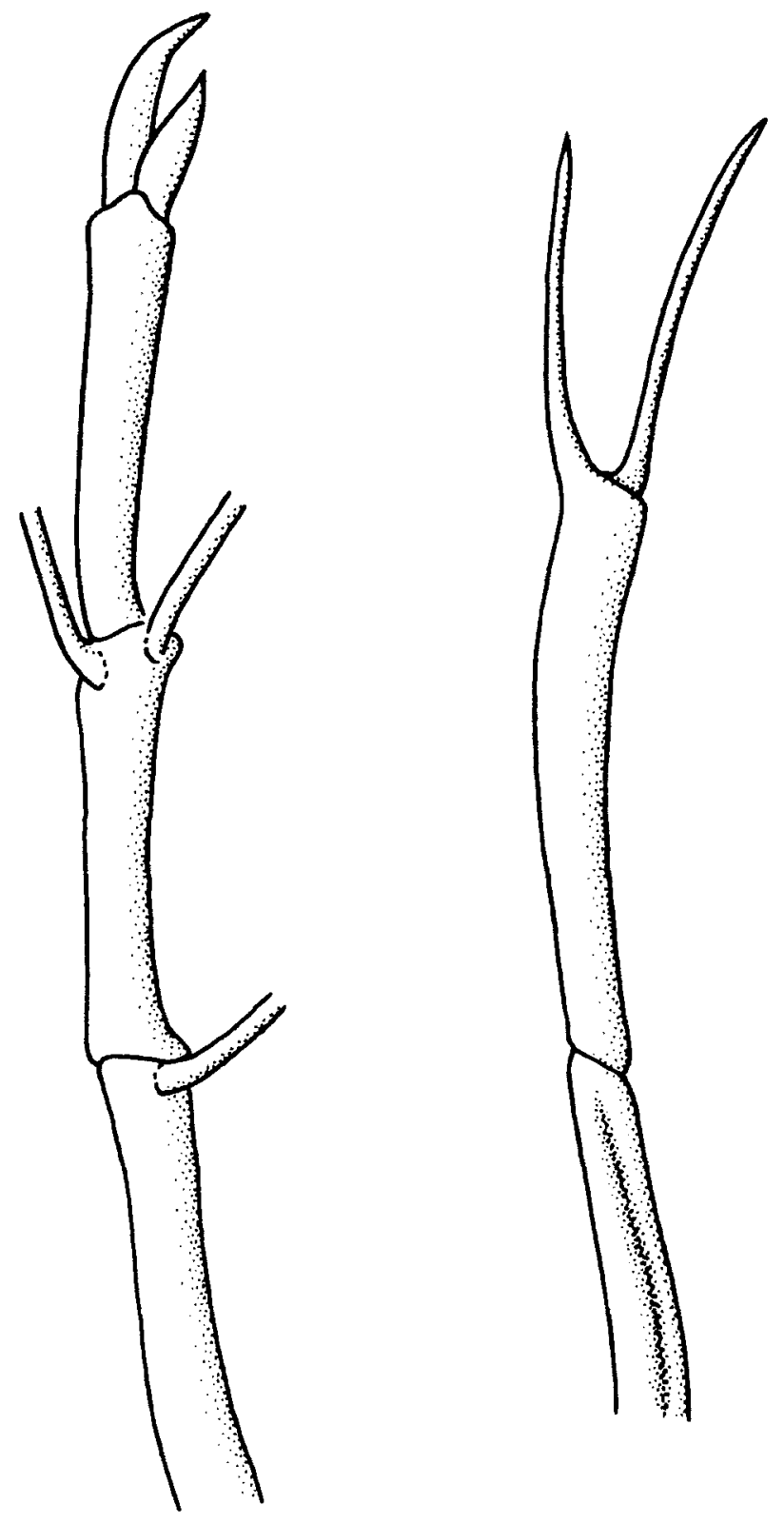

Abb. 3. rechts: Die letzten beiden Glieder eines Schreitbeins (Extremität II bis V) von Euproops mit langer "Endschere" (Post-Tarsus), äußerer Scherenteil gegen den starren inneren beweglich. links: Die letzten 3 Glieder eines ..Pushers" (Extremität VI) von Euproops. mit kleiner ...Endschere" (pincer) und stabähnlichen Fortsätzen an den Gelenkungen zwischen 5 . und 6 . sowie 6 . und 7 . Beinglied

Fig. 3. right: Leg of Euproops (extremities II to V). last two articulations with long claws (post-tarsus), outer part of claw movable. left: Leg (pusher) of Euproops (extremities VI). last three articulations with little pincer, with rod like processes at the joints

höchstens 1:3, während es bei Eluproops bis zu 1:7 ansteigen kann. An den jeweils etwas verbreiterten Gelenkungen kann zumindest ein kräftigerer Dorn nachgewiesen werden. Eine vergleichbare, wenn auch umfangreichere und kompliziertere "Bewehrung" weisen auch die Beine rezenter Limuliden auf (Gravier 1929. eigene Untersuchungen). Das letzte Beinglied ist sehr lang gestreckt und in zwei dünne Endglieder gespalten (,Spaltfuß“), die eine lange, schmale Schere bilden (Abb. 3 rechts, Taf. 1, Fig. 2).

Wie bei den rezenten Nachfahren läuft das Endglied beim „pusher" dagegen in eine deutlich kürzere Schere (,pincer") aus (Taf. 1, Fig. 5). An der Gelenkung zwischen 6.und 7. Beinglied entspringen mindestens zwei schmale, stabähnlich erhaltene Elemente. Ein weiteres, ähnliches Element findet sich auf der Innenseite der Gelenkung zwischen 5. und 6. Beinglied (Abb. 3 links). Diese Fortsätze dürften den paddelartigen Elementen in der gleichen Position bei den rezenten Limulidenbeinen entsprechen, wie sie Gravier (1929) bei Tachypleus tridentatus (Leach) beschreibt. Fisher (1979) gibt darüber hinaus noch an, dass Tibia und Patella getrennt sind, während sie bei modernen Limulaceen verwachsen sind. Das dürfte zu einer höheren Beweglichkeit der Gliedmaßen geführt haben. Nicht nachweisbar ist, ob der „pusher" 7 Glieder aufweist im Gegensatz zu den Extremitäten II bis $\mathrm{V}$. die jeweils nur 6 Glieder aufweisen, wie das bei den rezenten Limuliden der Fall ist (Gravier 1929).

\section{Beborstung}

Bei allen entsprechend gut erhaltenen Exemplaren von Euproops ist auf der Ventralseite ein dichter Besatz mit haarähnlichen Borsten nachweisbar. Dieser Besatz ist weitgehend auf die Ventralseite beschränkt und ist häufig nur sichtbar. wenn der Panzer weggebrochen ist, oder wenn das Tier mit der Ventralseite nach oben eingebettet wurde. Die Borsten erreichen Längen von $0,3 \mathrm{~mm}$ (Taf. 2, Figs $3,4,6$ ) und stehen mit einer Dichte von geschätzten annähernd 300 bis 400 pro $\mathrm{m}^{2}$ im Bereich der vermuteten Kiemen beieinander. Das Prosoma ist deutlich weniger dicht besetzt. Bei den juvenilen Formen sind auch die Randsaumstacheln am Außenrand mit kräftigen, gut sichtbaren Borsten besetzt (Taf. 2, Fig. 3), was man bei den Resten von adulten Tieren nicht mehr feststellen kann. Allerdings sind die Genal- und Intergenalstacheln nachweislich noch mit Borsten bewachsen, wenn auch deutlich weniger dicht, es finden sich auf der Unterseite eines Intergenalstachels nur noch etwa 100 Borsten auf den $\mathrm{mm}^{2}$ (Taf. 2, Fig. 6). Auch Anderson (1994) bildet bei der Rekonstruktion von Euproops danae bei einem adulten Exemplar eine lockere Beborstung der Genalstacheln 
sowie des Telsons ab. Wie die Borsten im restlichen ventralen Bereich der adulten Tiere verteilt sind, kann nicht gesagt werden, da kein entsprechend erhaltenes Material vorhanden ist.

\section{Deutung}

Viele Taxa der Ordnung Xiphosurida und dabei besonders der Unterordnung Bellinurina (im Sinne von Anderson \& Selden 1997) scheinen fast ausschließlich Formen hervorgebracht $\mathrm{zu}$ haben, die im terrestrisch-aquatischen und teilweise sogar subaerischen Bereich lebten. Für das Genus Euproops gibt es in der Literatur nur einen einzigen Beleg für eine Vergesellschaftung mit mariner Fauna. Copeland (1957) beschreibt diese Vergesellschaftung aus dem Oberkarbon von Kanada, vermutet aber eine Verschwemmung der Euproopiden in den marinen Bereich. Anderson (pers. Mitt., 2000) fand mehrfach Euproopiden in Schichten, die Sedimente mariner Ingressionen überlagern und vereinzelt Muscheln enthalten, deren Lebensraum als brackisch gedeutet wird. Im Zusammenhang damit vermutet Anderson (1997) eine offensichtlich bevorzugte Erhaltungsfähigkeit chitinöser Exoskelette unter brackischen Bedingungen,

Das höhere Westfal C und das bekannte Westfal D im Osnabrücker Raum gelten weitgehend als frei von marinen Einflüssen bis auf den Nachweis des Itterbeck-Horizontes anhand von einigen schlecht erhaltenen Agglutinatia (Knauff et al. 1971). Euproopiden sind dagegen im gesamten Profil nachweisbar, eine direkte Beziehung zu marinen Ingressionen kann nicht nachgewiesen werden. Auffällig ist jedoch das weitgehende Fehlen dieser in allen paralischen Becken häufig auftretenden Tiergruppe in den so genannten "intramontanen" Becken (Anderson 1997, Schultka 1988). Möglicherweise waren diese Tiere an eine Umwelt angepasst, die sich nur in ozeanisch beeinflusstem Klima entwickeln konnte.

Fisher (1979) geht ausführlich auf die Lebensweise der Euproopiden ein und kommt aufgrund sedimentologischer, paläobotanischer und funktionsmorphologischer Daten zu dem Schluss, dass die Euproopiden vornehmlich subaerisch lebten. Er geht sogar so weit, dass er gewisse Ähnlichkeiten des Exoskeletts bei Euproops danae mit Maiocercus sp. und Lycopsidenblättern als Mimikry und Phytomimese deutet. Diese Ansicht wird von Todd (1991) übernommen und auch
Brauckmann (1982) diskutiert diese Möglichkeit. Auf der Basis ausführlicher Untersuchungen an umfangreichem Material widerspricht Anderson (1994, 1997) dieser Meinung und weist auf Probleme mit der Physiologie und der Lokomotion hin. Auch Dunlop \& Horrocks (1996) lehnen eine so weitgehende Interpretation der oberflächlichen Ähnlichkeit von Euproops und Maiocercus ab. Diese beiden Autoren sprechen zudem von ganz unterschiedlichen Lebensräumen dieser beiden Gattungen (,aquatische Xiphosuren und terrestrische Arachniden"), Anderson (1997) hingegen schließt einen kurzzeitigen subaerischen Aufenthalt nicht aus.

Die dichte Beborstung der Euproopiden vom Piesberg, die die Ventralseite der Tiere bedeckt, belegt zumindest die Möglichkeit, dass die Euproopiden für bestimmte, kurze Zeitabschnitte subaerisch gelebt haben können. Dies gilt vor allem für juvenile Tiere, da bei ihnen die Beborstung besonders dicht ausgebildet ist. Damit dürften sie gerade in feuchter Laubstreu oder in Spülsäumen gute Lebensbedingungen gefunden haben, zumal sie hier vor ihren im Wasser lebenden natürlichen Feinden sicher waren (Möglicherweise wurde dieser Vorteil jedoch durch terrestrisch räuberisch lebende Feinde wieder aufgehoben.). Bei aquatischer Lebensweise ist die Ausbildung einer haarähnlichen Beborstung überflüssig. Daher bilden sich dichte Beborstungen bei aquatischen Tiergruppen gar nicht aus, oder sie bilden sich bei sekundär ins Wasser eingewanderten. Tiergruppen wieder zurück. Bei den rezenten Limuliden ist noch eine reliktäre Beborstung auf der Ventralseite, an den Extremitäten und am Panzerrand nachweisbar, durch die die Buchlungen feucht gehalten werden, wenn die Tiere für kurze Zeit das Wasser verlassen. Diese Funktion war bei Euproops aufgrund der relativen Länge und Dichte der Borsten erheblich effektiver, sodass diese Tiere die Möglichkeit hatten, länger subaerisch aktiv sein zu können. Durch die Länge der Borsten konnten sicherlich sogar Verfilzungen auftreten, was den wasserhaltenden Effekt noch verstärkt haben dürfte. Die Dichte und Verteilung der Borsten macht darüberhinaus eine Deutung als Tastorgane oder andere Sinnesborsten weniger wahrscheinlich.

Der schlanke Bau und die relativ langen Extremitäten mit den Fortsätzen an den vorderen Gelenken sprechen für eine Anpassung an laufende Fortbewegung auf instabilem, feinkörnigem Substrat (Anderson 1997), was mit und ohne Wasserüberdeckung gelten darf. Die Aus- 
bildung von Spaltfüßen könnte u.a. für greifende und klimmende Aktivitäten Vorteile bieten. Ausgewachsene Exemplare dürften allerdings weitgehend benthisch im Flachwasser gelebt haben und sind an diese Lebensbedingungen auch gut angepasst (Goldring \& Seilacher 1971). Für eine rein schwimmende Fortbewegung ist dieser Bau eher ungeeignet.

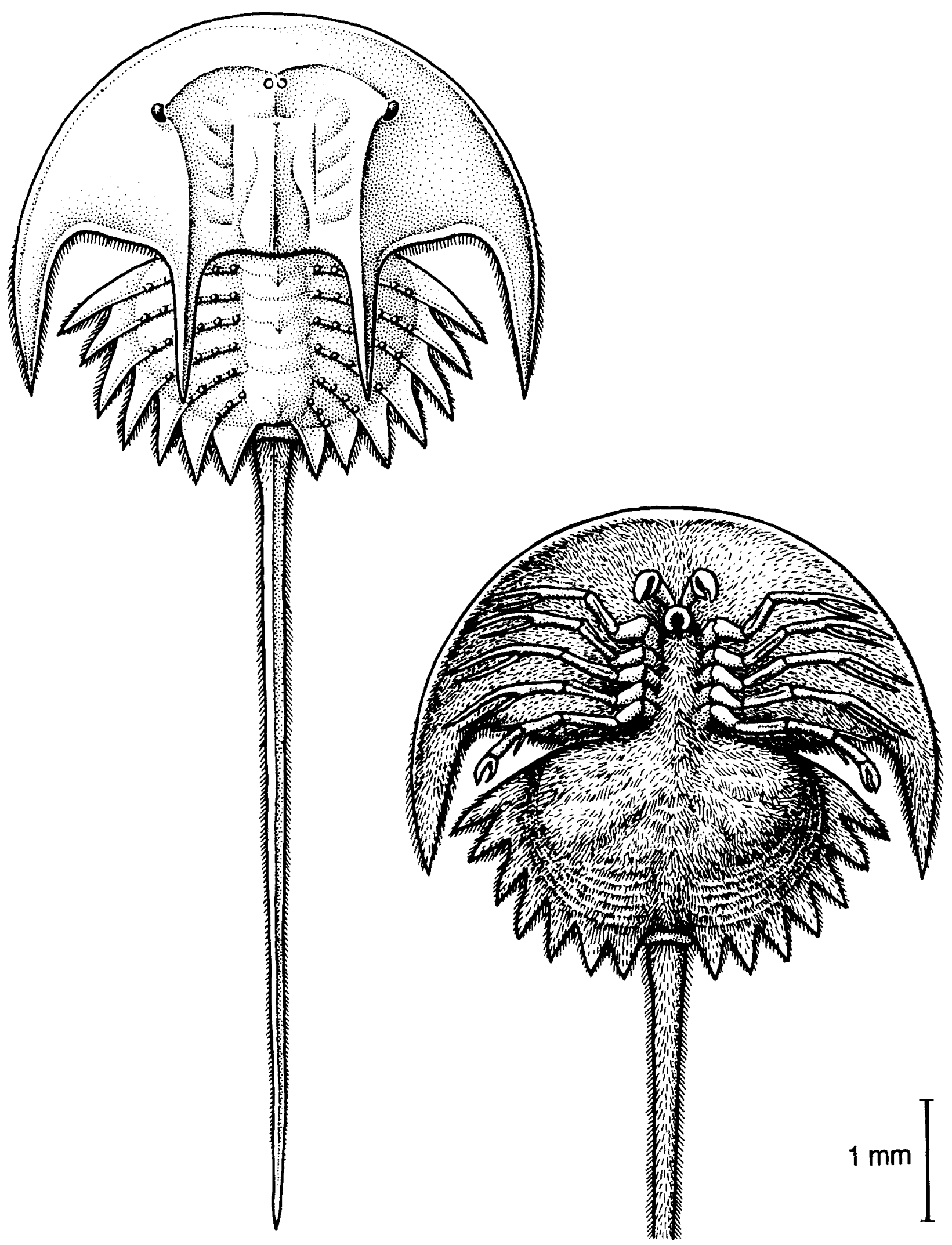

Abb. 4. Rekonstruktion eines juvenilen Individuums von Euproops mit der bauchseitigen Beborstung Fig. 4. Reconstruction of a juvenile Euproops, showing the setae of the ventral side 
Anderson (1997) führt die Zartheit der Extremitäten als Problem für die subaerische Fortbewegung an. Speziell für das Klettern auf Bäumen, wie das Fisher (1979) darlegt, sei der Bau ungeeignet. Angepasst an solch eine Lebensweise waren die Extremitäten sicherlich nicht, für kurzzeitige Aufenthalte und auch für das Überwinden von Hindernissen waren die Extremitäten aber sehr wohl geeignet (mdl. Mitt. O. Coleman, Museum für Naturkunde Berlin, 2000). Der Vergleich mit der Anpassung von subaerisch aktiven Crustaceen wie Birgus oder Grapsus (Konecky 1991, der fälschlicherweise die Limuliden zu den Krustaceen zählt), wird auch von Anderson (1997) abgelehnt.

Bei Auswertung der Informationen, die das Profil am Piesberg liefert, sowie der morphologischen Details, die über die Euproopiden des Westfal D bekannt geworden sind, kann man vermuten, dass diese Gruppe der Xiphosura während der ontogenetischen Entwicklung ihren Hauptlebensraum wechselten. Über die Larvalstadien wissen wir nichts, doch dürften sie - wie von den rezenten Vertretern dieser Tiergruppe bekannt - nektisch gelebt haben. Nach der ersten Häutung haben sie sich wahrscheinlich als Laubstreubewohner in gelegentlich trockenfallenden, gut durchlüfteten Spülsäumen am Rande sehr flacher, ruhiger Gewässer aufgehalten. Möglicherweise dienten ihnen sogar zeitweise Streuhorizonte am Boden der wassernahen Wälder als Lebensraum, zumindest hatten sie dafür die äußeren Voraussetzungen. Als Beleg dafür können die Funde juveniler Formen nur in stark FeOOH-schüssigen Sedimentabschnitten bzw. auf Schichtflächen gelten, die hauptsächlich mit einem einzigen Beblätterungstyp (Lycopsiden) ungeregelt bedeckt sind, was für einen Streuhorizont spricht. Die mehrfach beobachtete enge Beziehung von Lycopsiden-dominierten Streuhorizonten mit dem Auftreten von Euproopiden diskutieren bereits Fisher (1979) und Todd (1991).

Adulte Euproopidenreste finden sich dagegen vornehmlich in weitgehend pflanzenarmen Sedimenten, die nicht selten von Calamitenwurzeln durchzogen sind. Hier lebten die Tiere wahrscheinlich als Schlammwühler am Grunde der langsam verlandenden Seeränder, die mit Calamiten bestanden waren. Aber auch diesen Lebensraum konnten die Euproopiden gelegentlich, wenn auch nur für kürzere Zeitabschnitte, verlassen und bestimmte Strecken auf dem Festland zurücklegen, wie das Anderson (1994), Todd (1991) u.a. bereits angenommen haben.
Welche Funktion das im Verhältnis zur Körperlänge sehr lange (Taf. 1, Fig. 4) - aber auch gelenkige (Taf. 2, Fig. 1) - Telson in diesem Zusammenhang hatte, bzw. inwieweit das die Aktivitäten dieser Tiere im amphibischen Bereich behinderte, kann nicht gesagt werden.

\section{Danksagung}

Herr Dr. H. Kerp (Münster), Dr. Th. Rehren (Bochum) und Dr. H. W. J. van Amerom (ehem. Heerlen) gewährten mir großzügigen Zugang zu ihren Sammlungen. Herrn M. Born (München) bin ich in besonderer Weise für seine mehrtägige Sammlungstätigkeit zusammen mit mir auf der städtischen Müllhalde Osnabrück (Flöz Itterbeck) zu Dank verpflichtet. Dr. L. I. Anderson (Aberdeen), Dr. J. Dunlop (Berlin). Dr. C. Brauckmann (Clausthal-Zellerfeld) und Dr. O. Coleman (Berlin) danke ich für viele wertvolle Hinweise und die $\mathrm{Zu}$ gänglichmachung von Literatur. Frau W. Harre (Museum für Naturkunde, Berlin) ist für ihre unermüdlichen phototechnischen Bemühungen und Herrn J.-P. Mendau (Museum für Naturkunde, Berlin) sowie Frau J. Kauffeld (Berlin) für die Zeichenarbeiten sehr herzlich zu danken.

\section{Literatur}

Anderson, L. I. 1994. Xiphosurans from the Westphalian D of the Radstock Basin, Somerset Coalfield, the South Wales Coalfield and Mazon Creek, Illinois. - Proceedings of the Geologist's Association 105: 265-275.

- 1997. Taphonomy and Taxonomy of Palaeozoic Xiphosura. - Thesis submitted to the University of Manchester for the degreee of Ph.D. in the Faculty of Science. 151 pp., Selbstverlag, Manchester.

Anderson, L. I. \& Selden, P. A. 1997. Opisthosomal fusion and phylogeny of Palaeozoic Xiphosura. - Lethaia 30: $19-31$.

Bässler, R., Hoyer, P. \& Josten, K.-H. 1971. 3. Das Karbon von Ibbenbüren, am Hüggel und Piesberg. A) Stratigraphie. - Fortschritte in der Geologie von Rheinland und Westfalen 19: 75-78.

Bode, H. 1927. Palaeobotanisch-stratigraphische Studien im Ibbenbürener Carbon. - Abhandlungen der Preußischen Geologischen Landesanstalt, N. F. 106: 71 S.

Boekschooten, G. J. 1968. Ein neuer Fund eines Limulinen aus dem Osnabrücker Karbon. - Publicaties van het Natuurhistorisch Genootschap in Limburg 18 (3-4): 16.

Bölsche, W. 1875. Ueber die Gattung Prestwichia H. WOODW. und ihr Vorkommen in der Steinkohlenformation des Piesberges bei Osnabrück. - Jahresbericht des Naturwissenschaftlichen Vereins zu Osnabrück 2: $50-55$.

Brauckmann, C. 1982. Der Schwertschwanz Euproops (Xiphosurida, Limulina, Euproopacea) aus dem Ober-Karbon des Piesbergs bei Osnabrück. - Osnabrücker Naturwissenschaftliche Mitteilungen 9: 17-26.

- 1983. Ein Insektenrest (Odonata, Meganisoptera) aus dem Ober-Karbon des Piesberges bei Osnabrück. - Osnabrücker Naturwissenschaftliche Mitteilungen 10: 7-14.

- 1991. Einer neuer Insekten-Rest (Megasecoptera) aus dem Ober-Karbon von Osnabrück. - Osnabrücker Naturwissenschaftliche Mitteilungen 17: 25-32.

1995. Neue Insekten-Funde (Palaeodictyoptera: Breyeriidae) aus dem Ober-Karbon von Osnabrück (Deutschland). - Osnabrücker Naturwissenschaftliche Mitteilungen 20/21: 157-166. 
Brauckmann, C. \& Herd, J. 2000: Eine weitere neue Breveriiden-Art (Insecta: Palaeodictyoptera) aus dem OberKarbon von Osnabrück (Deutschland). - Neues Jahrbuch für Geologie und Paläontologie. Monatshefte 2000 (6): $333-344$.

Brauckmann, C., Gröning. E. \& Thiele-Bourcier, M. 1997. Kopf- und Schwanz-Region von Arthropleura armata Jordan, 1854 (Arthropoda: Ober-Karbon). - Geologica et Palaeontologica 31: 179-192.

Copeland, M. J. 1957. The Carboniferous Genera Palaeocaris and Euproops in the Canadian Maritime Provinces. Journal of Paleontology 31 (3): 595-599.

David, F. 1987. Sandkörper in fluviatilen Sandsteinen des Unteren Westfal D (Oberkarbon) am Piesberg bei Osnabrück. - Facies 17: $51-58$.

Dunlop, J. A. \& Horrocks. C. A. 1996. A new specimen of the Carboniferous trigonotarbid arachnid Maiocercus celticus (Pocock 1902) from Lancashire. UK. - Proceedings of the Yorkshire Geological Society 51 (1): 23-31.

Eagar, R. M. C., Bech. H.. Sommer. G. Sowiak. M. \& van Amerom, H. W. J. 1998. Neue Funde von nicht-marinen Muscheln im Steinbruch der Piesberger Steinindustrie $\mathrm{GmbH} \&$ Co KG am Piesberg (Westfal D) bei Osnabrück. - Osnabrücker Naturwissenschaftliche Mitteilungen 24: $31-38$.

Fisher. D. C. 1979. Evidence for subaerial activity of Euproops danae (Merostomata. Xiphosurida). In Nitecki. M. H. (ed.). Mazon Creek Fossils: 379-447. Academic Press. London.

Goldring, R. \& Seilacher. A. 1971. Limulid undertracks and their sedimentological implications. - Neues Jahrbuch für Geologie und Paläontologie. Abhandlungen 137: $422-442$.

Gothan. W. 1925. Ruhrkarbon und Osnabrücker Karbon. Glückauf 61: 777-778.

Gravier, Ch. 1929. Les Appendices Fouisseurs des Limules. - Archives d'Anatomie Microscopique 25: 270-279.

Haack. W. 1930. Der Piesberg bei Osnabrück. ein Bilderbuch der Erdgeschichte. - Teutoburger Wald und Weserbergland 4(11): $1-4$.

Hoffmann. F. 1826. Untersuchungen uber die Pflanzenreste des Kohlengebirges von Ibbenbüren und vom Piesberge bei Osnabrück. - Karsten's Archiv für Bergbau und Hüttenwesen 13 (2): 266-282

Josten, K.-H. 1966. Zur Flora des jüngeren Karbons (Westfal $\mathrm{C}$ bis Stefan) in Nordwestdeutschland und ihr Vergleich mit anderen Gebieten. Mit einem faunistischen Beitrag von E. Paproth. - Fortschritte in der Geologie von Rheinland und Westfalen 13 (1): 565-644.

Knauff, W., Köwing, K. \& Rabitz. A. 1971. Der erste Nachweis von Horizonten mit Foraminiferen im Westfal D von
Nordwestdeutschland. -- Fortschritte in der Geologie von Rheinland und Westfalen 18: 257-262.

Konecky. J. 1991. Tree-climbing horseshoe crabs. - Bulletin of the Southern Californian Paleontological Society 23: $101-102$.

Maithy. P. K. 1972. Fossil Flora of Westphalian D from Piesberg near Osnabrück. - Palaeontographica B 139 (5-6): $83-104$.

Meek. F. B. \& Worthen. A. H. 1865. Notice of some new types of organic remains from the Coal Measures of Illinois. - Proceedings of the Academy of Natural Sciences of Philadelphia 1865: 41-53.

Remy, W. 1975. The floral changes at the Carboniferous-Permian boundary in Europe and North America. In Barlow, J. A. (ed.). "The age of the Dunkard", Proceedings of the First I. C. White Memorial Symposium 1972: 305-352.

Roemer. F. A. 1860. Beiträge zur geologischen Kenntnis des nordwestlichen Harzgebirges. - Palaeontographica 9: $14-46$.

Schäfer, W. 1964. Aktuopaläontologische Beobachtungen, 2. Zur Fossilisation von Limulus. - Natur und Museum 94: $98-104$

Schultka, St. 1988. Beiträge zur Paläontologie der terrestren Räume unter besonderer Berücksichtigung des Ibbenbüren-Osnabrücker Karbons. - Inaugural-Dissertation an der Westfälischen Wilhelm-Universität Münster, 149 pp., Selbstverlag. Münster.

- 1994. Bellinurus cf. truemanii (Merostomata) aus dem tiefen Oberkarbon (Namur B/C) von Fröndenberg (Nordrhein-Westfalen. Deutschland). - Paläontologische Zeitschrift 68: $339-349$

Scott. A. C. 1979. The ecology of coal Measures floras from northern Britain. - Proceedings of the Geologist's Association 90 (3): $97-116$.

Siegfried. P. 1972. Ein Schwertschwanz (Merostomata, Xiphosurida) aus dem Oberkarbon von Ibbenbüren/Westf. Paläontologische Zeitschrift 46 (3-4): 180-185.

Sorusch. R. M. 1981. Untersuchungen zur Feinstratigraphie und Fazies im Karbon des Piesberges. Untersuchungen im Bereich Flöz Dreibänke. 81 pp., Münster. [unveröffentlicht]

Stœrmer, L. 1952. Phylogeny and Taxonomy of Fossil Horseshoe Crabs. - Journal of Paleontology 26 (4): 630-639.

- 1955. Merostomata, pp. 4-41. In Moore, R. C. (ed.) Treatise on Invertebrate Paleontology, Part P, Arthropoda 2: 181 pp.. University of Kansas Press, Lawrence.

Todd, J. A. 1991. A forest-litter animal community from the Upper Carboniferous?: notes on the association of animal body fossils with plants and lithology in the Westphalian D Coal Measures at Writhlington, Avon. - Proceedings of the Geologists' Association 102 (3): 179-184. 